\title{
A Spatial Coherence Approach to Minimum Variance Beamforming for Plane-Wave Compounding
}

\author{
Nghia Q. Nguyen and Richard W. Prager
}

\begin{abstract}
A new approach to implement minimum variance distortionless response (MVDR) beamforming is introduced for coherent plane-wave compounding. MVDR requires the covariance matrix of the incoming signal to be estimated and a spatial smoothing approximation is usually adopted to prevent this calculation from being under-constrained. In a new approach, we analyze MVDR as a spatial filter that decorrelates signals received at individual channels before summation. Based on the analysis, we develop two MVDR beamformers without using any spatial smoothing. In the first, MVDR weights are applied to the received signals after accumulating the data over transmits at different angles, while the second involves weighting the data collected in individual transmits and compounding over the transducer elements. In both cases, the covariance matrix is estimated using a set of slightly different combinations of the echo data. We show the sufficient statistic for this estimation can be described by approximating the correlation among the backscattered ultrasound signals to their spatial coherence. Using the van Cittert-Zernike theorem, their statistical similarity is assessed by relating the spatial coherence to the profile of the source intensity. Both spatial-coherence-based MVDR beamformers are evaluated on datasets acquired from simulation, phantom and in vivo studies. Imaging results show that they offer improvements over simple coherent compounding in terms of spatial and contrast resolution. They also outperform other existing MVDR-based methods in the literature that are applied to coherent plane-wave compounding.
\end{abstract}

Index Terms - adaptive beamforming, coherent planewave compounding, minimum variance beamforming, spatial coherence, van Cittert-Zernike theorem, acoustic reciprocity, image quality.

\section{INTRODUCTION}

In array processing, a beamformer is defined as a spatial filter associated with a sensor array to receive a signal arriving at a specific location while attenuating those radiating at the other locations [1]. When applied to ultrasound imaging, this filter involves a set of delays and weights that are applied to each transducer element to control the acoustic beam shape on transmit and a similar set that are applied on receive to create focal zones [2]. The goal is to generate a uniformly narrow pulse-echo beam with low sidelobe amplitudes over the entire imaging region. This enables the reconstruction of a B-scan image with high contrast and spatial resolution [3]. The beamformer performance, therefore, can be evaluated by using these figures of merit, along with the echo signal-to-noise ratio (eSNR) that characterizes the beam penetration [4].

Over the last decade, minimum variance distortionless response (MVDR) beamforming has been comprehensively investigated by many ultrasound imaging groups [5]-[8], as an alternative to conventional delay-and-sum (DAS) beamforming. The MVDR beamformer generates imaging data in a manner that minimizes the interference and noise while maintaining the signals reflected from the main target. The beamforming weights can be calculated directly from inversion of the data covariance matrix. This type of approach is classified as adaptive beamforming implying the beamformer is optimized based on the incoming signals.

In practice, MVDR performance is mainly limited by errors in the covariance matrix estimation. These errors are unavoidable especially in a conventional acquisition system where data at each imaging point is generated from only one pulse-echo sequence. As a result, the covariance matrix is estimated from only one vector sample of the signals, or snapshot, collected at the receive aperture. If the errors are too large, the MVDR-based algorithm quickly degrades the image quality to a point where it is even worse than conventional DAS. Several approximations have been proposed to overcome this issue. Most popular among them is spatial smoothing that reduces the aperture size so that several realizations of the data vector can be formed over the receive array [8]. To avoid illconditioning, the number of snapshots should be greater than or equal to the matrix size [9]. This limits the aperture size to less than or equal to half the original vector length. Although spatial smoothing helps improve the beamformer robustness, this reduction of the effective aperture significantly decreases the image resolution.

Developments in hardware flexibility make it realistic to implement two-way focussing ultrasound imaging systems, i.e. the pulse-echo beam is generated with both transmit and receive focussing over the entire imaging region [3]. Such systems include synthetic aperture imaging [10], coherent plane-wave compounding [11], and pixel-based beamforming with focused beams [12], [13]. In these approaches, the transmit focusing is synthesized by accumulating data from a large number of pulse-echo sequences. This process creates more echo data that can be used for the covariance matrix estimation, and hence can enhance the MVDR performance. Yet, most MVDR beamformers applied to these systems still use the spatial smoothing approximation [14]-[17], which compromises the image quality.

In [18], Wang et al. proposed an MVDR beamformer based on a synthetic aperture approach, without using spatial smoothing. They estimated the covariance matrix using data vectors acquired from several different transmits; they also used a 
diagonal loading parameter to regularize the calculation of the inverse. The resulting matrix was named the pseudocovariance due to the fact that the data for the calculation are not generated using a single transmission. The method is faster than using the spatial smoothing approach but was found to compromise the eSNR of the generated images [19].

In this paper, we develop new MVDR-based algorithms for coherent plane-wave compounding (CPWC) by upgrading the pseudocovariance matrix calculation. Compared to other MVDR studies in the ultrasound literature, the novelty of our approach is to consider the beamformer as a spatial filter used to decorrelate the echo data as in array signal processing [20]. The signal correlation is approximated to the spatial coherence, a measure of the similarity of backscattered echoes received on different transducer elements [21]. Based on the analysis, we propose two MVDR beamformers; they are each applied to a differently constituted vector of compounded data. The first is performed on the superposition of the received data from multiple transmits, where each transmit involves an outgoing wave at a different angle; so we accumulate across the transmits and then apply the MVDR weights to the different transducer elements. The second is applied to postsummed signals within individual transmits; so we accumulate across the receive elements and apply the MVDR weights to the different transmit angles. In each beamformer, the snapshots for the covariance matrix estimation are generated by combining a set of vectors, formed from slightly different combinations of the echo data. The statistical similarity between each input vector and its corresponding snapshots is explained using the van Cittert-Zernike theorem, developed for ultrasound imaging [22]. This links the spatial coherence among signals to the Fourier transform of the source pattern. Our proposed beamformers are demonstrated on a series of datasets acquired using a research scanner [23]. They are also demonstrated in an in vivo study where the data has more realistic clinical characteristics.

The rest of the paper is organized as follows. In Section II, we describe a standard MVDR beamformer as a spatial filter, applied across the transducer aperture. Then we review two recent MVDR beamformers applied to CPWC [16], [17], as well as the pseudocovariance matrix calculation in [18]. In Section III, we develop two new MVDR beamformers based on the spatial coherence approach. All beamformers are then demonstrated on datasets obtained from simulation, phantom, and in vivo studies in Section IV. Their performance is evaluated based on the spatial and contrast resolution of the generated images. The results are discussed and explained in Section V. Finally, we summarize our work in Section VI.

\section{LiterATURE REVIEW}

\section{A. MVDR Beamforming as a Spatial Filter}

We begin by introducing a standard MVDR beamformer applied to conventional ultrasound imaging [8]. That is, it generates an image line-by-line; after each transmit the backscatter down the centreline of that transmit is estimated. For convenience, we denote the imaging data and its associated vector dimensions using a common notation convention. This notation is extended in the next section when we describe MVDR beamforming for coherent plane-wave compounding.

Consider a receive aperture of $N$ elements and let $\mathbf{x}(k)$ be a data vector collected from the aperture for an imaging point $\mathrm{P}$ at time instant $n, \mathbf{x}(n)=\left[x_{1}(n), x_{2}(n), \ldots, x_{N}(n)\right]^{\mathrm{T}}$. The vector can be modeled as

$$
\mathbf{x}(n)=s_{p}(n) \mathbf{a}\left(\tau_{p}\right)+\mathbf{i}(n),
$$

where $s_{p}(n)$ is the signal waveform at $\mathrm{P}, \mathbf{i}(n)$ represents a combination of the acquisition noise and off-target interference, $\mathbf{a}\left(\tau_{p}\right)$ is the time-delay vector.

The beamforming weight vector $\mathbf{w}$ is calculated by [20]

$$
\mathbf{w}=\frac{\mathbf{R}_{i}^{-1} \mathbf{a}\left(\tau_{p}\right)}{\mathbf{a}^{H}\left(\tau_{p}\right) \mathbf{R}_{i}^{-1} \mathbf{a}\left(\tau_{p}\right)},
$$

where $\mathbf{R}_{i}$ is the $N \times N$ interference-plus-noise covariance matrix, and $(\cdot)^{H}$ stands for the Hermitian transpose. The beamformer output is obtained as

$$
y(n)=\mathbf{w}^{H} \mathbf{x}(n)=\sum_{m=1}^{N} w_{m}^{*} x_{m}(n) .
$$

Equation (2) is derived under the assumption of narrowband signals for the radio-frequency (RF) data. It assumes the backscattered echoes arriving at individual transducer receive channels are only different in their phase components, or in the time delays from the imaging point to each of these channels [24]. Although this is a poor approximation for ultrasonic signals, we adopt the assumption for the sake of simplicity. As there is similarity or correlation between each pair of echoes received on transducer elements when backpropagated from a point-scatterer [21], the MVDR acts as a spatial filter that suppresses noise and off-target interference by decorrelating them before adding them together. Through the matrix inversion lemma, one can show that the decorrelation is still equivalent if it is applied to all components of $\mathbf{x}(k)$, from which $\mathbf{w}$ is given by [20]

$$
\mathbf{w}=\frac{\mathbf{R}_{x}^{-1} \mathbf{a}\left(\tau_{p}\right)}{\mathbf{a}^{H}\left(\tau_{p}\right) \mathbf{R}_{x}^{-1} \mathbf{a}\left(\tau_{p}\right)},
$$

where $\mathbf{R}_{x} \triangleq \sigma_{p}^{2} \mathbf{a}\left(\tau_{p}\right) \mathbf{a}^{H}\left(\tau_{p}\right)+\mathbf{R}_{i}$ - the data covariance matrix, and $\sigma_{p}^{2}$ is the scattering strength at $\mathrm{P}$. The main signal $s_{p}(k)$ is preserved by matching the filter output to $\mathbf{a}\left(\tau_{p}\right)$. This equation allows the MVDR to be implemented directly on $\mathbf{x}(k)$. By assuming $\mathbf{a}\left(\tau_{p}\right)$ is known exactly through a constant sound-speed $(c=1540 \mathrm{~m} / \mathrm{s})$, the beamformer performance depends on the accuracy with which it is possible to determine $\mathbf{R}_{x}$.

Since there is only one sample vector of $\mathbf{x}$ available, an approximation is needed to estimate $\mathbf{R}_{x}$. In the most popular method, called spatial smoothing, $\mathbf{x}(n)$ is divided into $N-$ $L+1$ overlapping vectors of length $L$, and $\mathbf{R}_{x}$ is estimated as

$$
\widehat{\mathbf{R}}_{x}=\frac{1}{N-L+1} \sum_{l=1}^{N-L+1} \mathbf{x}_{l}(n) \mathbf{x}_{l}^{H}(n),
$$

where $\mathbf{x}_{l}(n)=\left[x_{l}(n), x_{l+1}(n), \ldots, x_{l+L-1}(n)\right]^{T}-$ the $l^{\text {th }}$ subarray formed over $\mathbf{x}(n)$. 

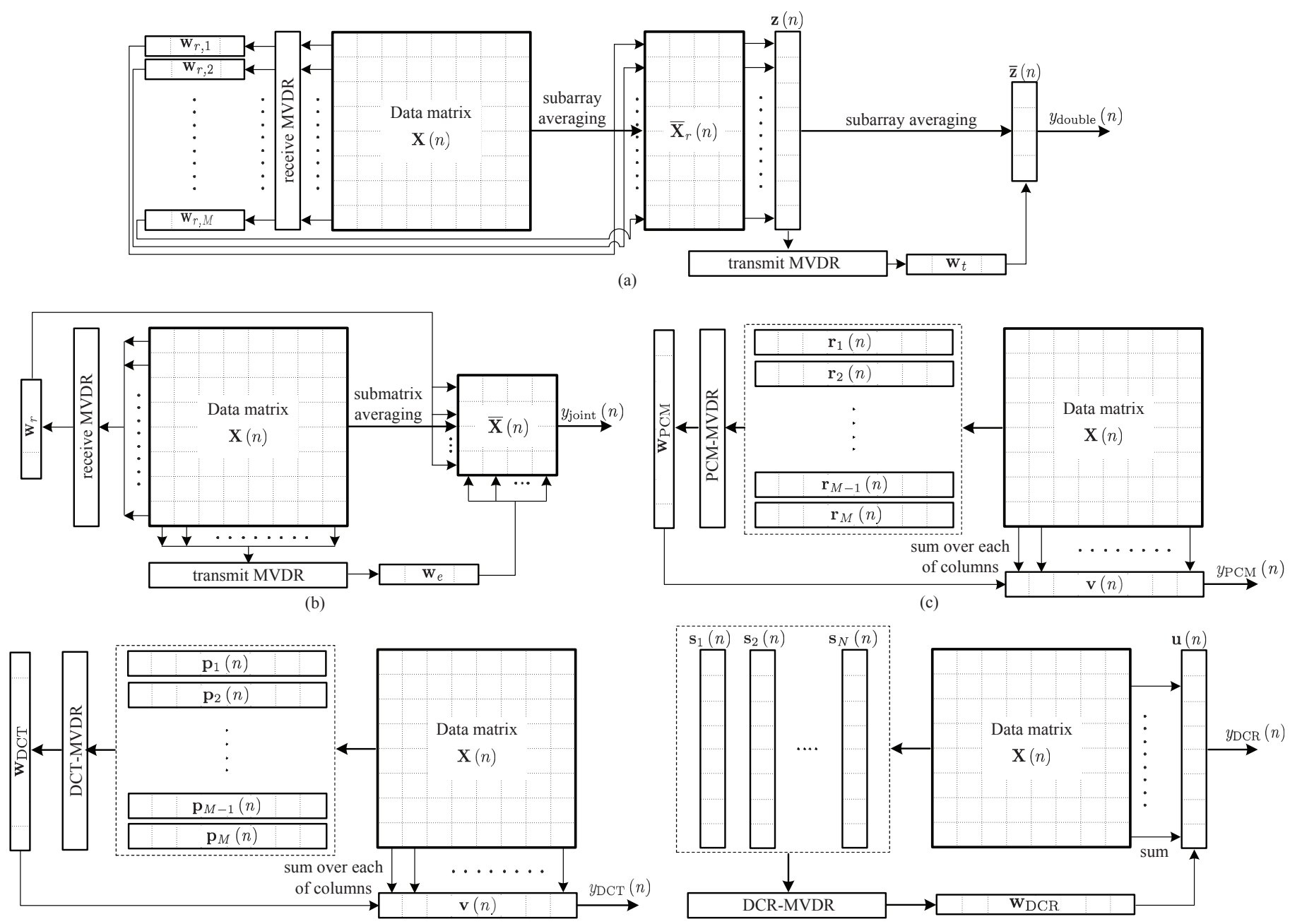

(d)

(e)

Fig. 1. Diagrams of the MVDR beamformers from data matrix $\mathbf{X}(n)$ to the beamformed output signals: (a) double MVDR, (b) joint MVDR, (c) PCM-MVDR, (d) DCT-MVDR, and (e) DCR-MVDR. The transmit and receive MVDR beamformers in (a) and (b) are calculated with the spatial smoothing approximation.

As a result of the spatial smoothing approximation, $w$ has its size reduced to $L$. Thus, $\mathbf{x}(n)$ also needs to be adapted to match this new length. It is modified to $\overline{\mathbf{x}}(n)$ through subarray averaging, given by

$$
\overline{\mathbf{x}}(n)=\frac{1}{N-L+1} \sum_{l=1}^{N-L+1} \mathbf{x}_{l}(n)
$$

For $\widehat{\mathbf{R}}_{x}$ to be nonsingular, the number of snapshots $N-L+1$ should be greater than or equal to the matrix size $L$, or $L \leq$ $(N+1) / 2$ [9]. To enhance the beamformer robustness, the estimation is also combined with a diagonal loading technique. This adds a small constant $\varepsilon$ to the elements on the main diagonal of $\widehat{\mathbf{R}}_{x}$, i.e., the estimated matrix becomes $\widehat{\mathbf{R}}_{x}+\varepsilon \mathbf{I}$. Using a smaller subarray length or increasing parameter $\varepsilon$ enhances the non-singularity of the estimated matrix but at greater cost to the image resolution. This reduces the benefit of the MVDR beamforming and makes it closer in terms of performance to a DAS beamformer.

\section{B. Coherent Plane-wave Compounding}

In CPWC, data are acquired by insonifying with planewave beams in multiple directions over the entire imaging region. The travel time delay depends on the angle of each pulse. Details of the calculations are provided in [11]. By assuming the transmit beam is steered in $M$ different angles and backscattered waveforms are received on an $N$-element linear array, the signals with time index $n$, after appropriate delays for the specific pixel under consideration, can be arranged in a 2-D matrix $\mathbf{X}(n)$ of $M \times N$, given by [17]

$$
\mathbf{X}(n)=\left[\begin{array}{cccc}
x_{1,1}(n) & x_{1,2}(n) & \cdots & x_{1, N}(n) \\
x_{2,1}(n) & x_{2,2}(n) & \cdots & x_{2, N}(n) \\
\vdots & \vdots & \ddots & \vdots \\
x_{M, 1}(n) & x_{M, 2} & \cdots & x_{M, N}(n)
\end{array}\right]
$$

where $x_{i j}(n)$ is the signal received on channel $j$ after a transmitted excitation at firing angle $i$. By averaging all of these matrix elements, we obtain the compounded data as

$$
y_{\mathrm{CPWC}}(n)=\frac{1}{M N} \sum_{i=1}^{M} \sum_{j=1}^{N} x_{i j}(n) \text {. }
$$


MVDR beamforming can be integrated into this compounding strategy by being applied to the rows or the columns of the matrix. The former option involves applying it to data within each plane-wave transmission, while in the latter option it is applied across the different firing angles, on the signals collected from one element or on combined signals from several transducers. To differentiate these options from each other, we name them the receive element weighting MVDR (or receive MVDR) and transmit beam weighting MVDR (or transmit MVDR), respectively. In the next two sections, we describe two MVDR-based algorithms applied to the CPWC in [16], [17]. They can be viewed as compositions of the transmit and receive MVDRs in different ways. All involve covariance matrices calculated using the spatial smoothing approximation.

\section{Double MVDR beamformer}

The MVDR approach can be applied as a receive beamformer to each row of the $\mathbf{X}(n)$ matrix. It can then be re-used as a transmit beamformer to combine the results. This strategy forms the double MVDR [16].

First, let $\mathbf{r}_{i}(n)=\left[x_{i, 1}(n), x_{i, 2}(n), \ldots, x_{i, N}(n)\right]^{T}-\mathbf{a}$ vector transposed from row $i$. It includes receive signals acquired from transmit event $i$. By applying the receive MVDR to $\mathbf{r}_{i}(n)$, we calculate the weight vector $\mathbf{w}_{r, i}(r:$ receive $)$ and obtain $z_{i}(n)$ - the beamforming output at row $i$. In the second step, all calculated $z_{i}(n)$ are stacked together to form an $M$ dimensional vector $\mathbf{z}(n)$. The transmit MVDR is applied to $\mathbf{z}(n)$ to obtain the beamforming weight vector $\mathbf{w}_{t}(t$ : transmit) and the overall output $y_{\text {double }}(n)$. The diagram for the double MVDR beamformer is shown in Fig. 1(a). In the diagram, $\overline{\mathbf{X}}_{r}(n)$ is the modification of $\mathbf{X}(n)$ after applying subarray averaging to all of its rows, and $\overline{\mathbf{z}}(n)$ is the subarray-averaged version of $\mathbf{z}(n)$. These modifications are required to adapt to the needs of the spatial smoothing approximations used to estimate the covariance matrices. In each receive MVDR applied to $\mathbf{r}_{i}(n)$, the covariance matrix estimation includes the diagonal loading term of $\varepsilon_{r, i} \mathbf{I}$. Similarly in the transmit MVDR applied to $\mathbf{z}(n)$, the estimation includes the diagonal loading term of $\varepsilon_{t} \mathbf{I}$.

\section{Joint MVDR Beamformer}

In [17], Zhao et. al. proposed the joint MVDR beamformer where the transmit and receive beamformers are applied to $\mathbf{X}(n)$ simultaneously. The diagram is shown in Fig. 1(b). In the joint MVDR, we denote $\mathbf{w}_{r}$ ( $r$ : receive) as the receive element weight vector. These weights may well be different from the $\mathbf{w}_{r, i}$ weights in the double MVDR. Similarly, we denote $\mathbf{w}_{e}(e$ : emission) as the transmit beam weight vector which can also be different from $\mathbf{w}_{t}$. Both $\mathbf{w}_{r}$ and $\mathbf{w}_{e}$ are calculated directly on the pre-summed data. This strategy has advantages in the context of implementation on parallel computing architectures. However this is beyond the scope of the present study.

The matrix for calculating the receive weight $\mathbf{w}_{r}$ is given by

$$
\widehat{\mathbf{R}}_{r}=\frac{1}{M} \sum_{i=1}^{M} \widehat{\mathbf{R}}_{r, i}+\varepsilon_{r} \mathbf{I}
$$

where $\widehat{\mathbf{R}}_{r, i}$ is the estimated covariance matrix of $\mathbf{r}_{i}(n)$ from the use of the spatial smoothing approximation, $\varepsilon_{r}$ is the diagonal loading parameter. We recall that $\mathbf{r}_{i}(n)$ is a column vector of received signals acquired in transmit event $i$.

Similarly, the transmit weight $\mathbf{w}_{e}$ has the corresponding matrix estimation as follows:

$$
\widehat{\mathbf{R}}_{e}=\frac{1}{N} \sum_{j=1}^{N} \widehat{\mathbf{R}}_{e, j}+\varepsilon_{e} \mathbf{I}
$$

where $\widehat{\mathbf{R}}_{e, j}$ is the estimate covariance matrix of the $j^{\text {th }}$ column $\mathbf{e}_{j}(n)$ of $\mathbf{X}(n)$ using spatial smoothing, i.e., $\mathbf{e}_{j}(n)=$ $\left[x_{1, j}(n), x_{2, j}(n), \ldots, x_{M, j}(n)\right]^{T}$ - a vector of signals received on element $j$ and collected over all transmit events, $\varepsilon_{e}$ is the diagonal loading parameter. The matrices $\widehat{\mathbf{R}}_{r}$ and $\widehat{\mathbf{R}}_{e}$ are inverted in order to calculate $\mathbf{w}_{r}$ and $\mathbf{w}_{e}$, respectively.

The spatial smoothing approximations reduce the sizes of $\mathbf{w}_{r}$ and $\mathbf{w}_{e}$. Thus, $\mathbf{X}(n)$ needs to be modified before calculating the beamformer output. The modified matrix $\overline{\mathbf{X}}(n)$ is created from $\mathbf{X}(n)$ using submatrix averaging. The full expression for $\overline{\mathbf{X}}(n)$ is provided in Eq. (11) at the top of Page 5 . The beamformed output is given by

$$
y_{\text {joint }}(n)=\mathbf{w}_{e}^{H} \overline{\mathbf{X}}(n) \mathbf{w}_{r} .
$$

The joint MVDR has two major differences from the double MVDR. First, its transmit weight $\mathbf{w}_{e}$ is calculated directly on the pre-summed echo data, while in the double MVDR, $\mathbf{w}_{t}$ is calculated based on data that has been through the receive MVDR beamformer. Second, the receive weight $\mathbf{w}_{r}$ in the joint MVDR is guaranteed to be the same when applied to data from all transmits, while $\mathbf{w}_{r, i}$ could be different from one transmit to another.

\section{E. Pseudo-Covariance-Matrix MVDR Beamformer}

Before proposing our MVDR beamformers for CPWC, we summarize the pseudocovariance matrix of the MVDR beamformer applied to synthetic aperture imaging in [18]. Our first beamformer, described in Section III-A, is developed by enhancing the calculation of this matrix.

The pseudocovariance matrix is calculated in a similar way to (9) but without using spatial smoothing:

$$
\widehat{\mathbf{R}}_{\mathrm{PCM}}=\frac{1}{M} \sum_{k=1}^{M} \mathbf{r}_{k}(n) \mathbf{r}_{k}^{H}(n)+\varepsilon \mathbf{I} .
$$

The beamforming weights are calculated by inverting $\widehat{\mathbf{R}}_{\text {PCM }}$, similar to Eq. (4). They are used to apodize data received within each of the transmits. The results are then superposed to obtain the beamformer output. We adopt this strategy to form a new MVDR beamformer applied to CPWC. We refer to it as the pseudocovariance-matrix MVDR (PCMMVDR). Its diagram is shown in Fig. 1(c). The PCM-MVDR can be considered as an extension of the joint MVDR where $\mathbf{R}_{r}$ is estimated without using spatial smoothing and $\mathbf{w}_{e}$ is simply a uniform vector. 


$$
\overline{\mathbf{X}}(n)=\frac{1}{\left(N-L_{1}+1\right)\left(M-L_{2}+1\right)} \sum_{i=1}^{M-L_{2}+1} \sum_{j=1}^{N-L_{1}+1}\left[\begin{array}{cccc}
x_{i, j}(n) & x_{i, j+1}(n) & \cdots & x_{i, j+L_{1}-1}(n) \\
x_{i+1, j}(n) & x_{i+1, j+1}(n) & \cdots & x_{i+1, j+L_{1}-1}(n) \\
\vdots & \vdots & \ddots & \vdots \\
x_{i+L_{2}-1, j}(n) & x_{i+L_{2}-1, j+1} & \cdots & x_{i+L_{2}-1, j+L_{1}-1}(n)
\end{array}\right]
$$

\section{Spatial Coherence Approach to Minimum VARIANCE BEAMFORMING}

\section{A. Data-Compounded-among-Transmit MVDR Beamformer}

First, we realize that the PCM-MVDR output remains unchanged if we apply $\mathbf{w}_{\mathrm{PCM}}$ after data superposition among all rows of $\mathbf{X}(n)$. Alternatively, the input vector of PCM-MVDR can be considered as a data vector compounded over multiple transmits. We denote it by $\mathbf{v}(n)=$ $\left[v_{1}(n), v_{2}(n), \ldots, v_{N}(n)\right]^{T}$ where

$$
v_{j}(n)=\sum_{i=1}^{M} x_{i, j}(n) \text { for } j=\overline{1, N},
$$

which is the summed signals on element $j$ over all transmit events. We recall that $(i, j)$ are used respectively to index $M$ transmit events and $N$ receive elements.

By considering the MVDR beamforming as a spatial filter, we wish to decorrelate the input vector $\mathbf{v}(n)$ by using a matrix that is equal or close to the second-order statistics of $\mathbf{v}(n)$. Matrix $\widehat{\mathbf{R}}_{\mathrm{PCM}}$, however, is estimated from $\mathbf{r}_{k}$, each of which is a small part of $\mathbf{v}(n)$ and unrelated to the others. To achieve better decorrelation, we would like to create new snapshots of data with the second-order statistics similar to that of $\mathbf{v}(n)$. Thus, we generate a new set of snapshot $\mathbf{p}_{k}(n)=$ $\left[p_{k, 1}(n), p_{k, 2}(n), \ldots, p_{k, N}(n)\right]^{T}$, where

$$
p_{k, j}(n)=\frac{1}{M-1} \sum_{\substack{i=1 \\ i \neq k}}^{M} x_{i, j}(n) .
$$

Vector $\mathbf{p}_{k}(n)$ is still composed of compounded data like $\mathbf{v}(n)$ but excluding echo signals from transmit event $k$. The statistical similarity between $\mathbf{v}(n)$ and $\mathbf{p}_{k}(n)$ can be described as follows. Take $\mathbf{v}(n)$ first. It comprises coherently compounded signals among multiple planar transmits. In [11], Montaldo et al. showed that such compounding is equivalent to the insonification of the focused beam at its focal point, where the beam is a combination of plane-wave beams from $M$ directions. By approximating each plane wave to a spherical pulse, we can assume the beam is generated from an $M$ element pseudo phased array. This argument is valid for every pixel in the imaging region. By approximating the covariance matrix of the signals in $\mathbf{v}(n)$ to the spatial coherence among them, we can apply the van Cittert-Zernike (VCZ) theorem to relate its statistics to the profile of the source intensity. The VCZ theorem describes the spatial coherence between the two received waveforms observed at two separate points $\mathrm{P}_{1}$ and $\mathrm{P}_{2}$. It is equal to the Fourier transform of the source pattern taken at the spatial frequency that relates to the distance between $P_{1}$ and $P_{2}$ (see [25], Chap. 5). In this case, $P_{1}$ and $\mathrm{P}_{2}$ are the positions of the receiving transducer elements.
Similarly, the covariance matrix of $\mathbf{p}_{k}(n)$ approximates the spatial coherence among its components, which is determined by the beam generated from the same $M$-element pseudo phased array, but with element $k$ deactivated. This deactivation is the only difference when comparing the spatial coherence of $\mathbf{v}(n)$ to that of $\mathbf{p}_{k}(n)$. For a large number, $M$, of transmits, we assume this variation is small enough so that the vectors have similar second-order statistics to each other. This explains how the covariance matrix of $\mathbf{v}(n)$ can be better estimated by using snapshots $\mathbf{p}_{k}(n)$.

The VCZ theorem was derived for ultrasound imaging in [22]. In that work, the second-order statistics of the data was calculated in terms of the spatial covariance, which includes normalizing terms. In our study, the covariance matrix does not include any normalizing term. Each of its elements is more similar to the spatial coherence in statistical optics [25], or the visibility in radio-astronomy imaging (see [26], Chap. 5).

Over the entire range of firing angles, we can form $M$ such vectors of $\mathbf{p}_{k}(n)$ for the matrix estimation $(k=\overline{1, M})$. The covariance matrix can then be estimated:

$$
\widehat{\mathbf{R}}_{\mathrm{DCT}}=\frac{1}{M} \sum_{k=1}^{M} \mathbf{p}_{k}(n) \mathbf{p}_{k}^{H}(n)+\varepsilon \mathbf{I},
$$

where $\varepsilon$ is the diagonal loading parameter.

This estimation forms a new strategy to calculate the covariance matrix for the MVDR beamforming. As the snapshots are generated by using data compounded among multiple transmits, we name it data-compounded-among-transmit $M V D R$ (DCT-MVDR). A diagram of the beamformer is shown in Fig. 1(d).

\section{B. Data-Compounded-on-Receive MVDR Beamformer}

We propose another beamformer using the spatial coherence approach based on the foregoing analysis. The beamformer is applied to a vector composed of data superposed over the echo signals received within individual transmits. By denoting it as $\mathbf{u}(n)=\left[u_{1}(n), u_{2}(n), \ldots, u_{M}(n)\right]^{T}$, each of its elements, $u_{i}(n)$, is given by

$$
u_{i}(n)=\sum_{j=1}^{N} x_{i, j}(n) \text { for } i=\overline{1, M} .
$$

In our analysis, we describe the MVDR beamformer as a filter that suppresses the spatial coherence among echo signals. The spatial coherence occurs between two signals propagated from the same point-source [25]. In ultrasound imaging, however, the source is reflected from the transmit beam. Thus, a MVDR beamformer should be applied to decorrelate echo signals within one transmission. The input vector $\mathbf{u}(n)$, however, has components generated in different 
transmits. We can still view this MVDR beamformer as a decorrelation by making use of the acoustic reciprocity theorem that exchanges the roles of transmit and receive in the context of spatial coherence [27]. From that perspective, the post-summed signals in $\mathbf{u}(n)$ can be considered as echo data received on the $M$-element pseudo-phased-array when the beam is insonified from an $N$-element linear array. The MVDR beamformer is thus used to decorrelate the signals coming from these $M$ directions.

Similar to the DCT-MVDR beamforming, we generate multiple snapshots $\mathbf{s}_{k}(n)$ to represent different aspects of $\mathbf{u}(n)$ by dropping the echo data from transducer element $k$ out of the superposition. This has $i^{\text {th }}$ component, $s_{k, i}(n)$, given by

$$
s_{k, i}(n)=\sum_{\substack{j=1 \\ j \neq k}}^{N} x_{i, j}(n) .
$$

Over the entire $N$-element array, we can generate $N$ snapshots for the covariance matrix estimation. Because of the dynamic focusing of CPWC on receive, acoustic reciprocity means that we can consider each of $\mathbf{s}_{k}(n)$ and $\mathbf{u}(n)$ as being composed of backscattered signals from the foci of the corresponding beams. These beams are generated from the linear array of $N$ elements. Thus the VCZ theorem can be applied to describe the statistical similarity between $\mathbf{u}(n)$ and each of $\mathbf{s}_{k}(n)$, in a similar way to the argument above for the DCT-MVDR beamformer. For the DCT-MVDR, we discussed how the spatial frequency in the coherence function is determined by the distance between the two transducer positions $\mathrm{P}_{1}$ and $\mathrm{P}_{2}$. For the present algorithm, acoustic reciprocity tells us that this distance must now be replaced by a parameter $\Delta \Theta$, the difference between the two steering angles.

The covariance matrix is estimated using

$$
\widehat{\mathbf{R}}_{\mathrm{DCR}}=\frac{1}{N} \sum_{k=1}^{N} \mathbf{s}_{k}(n) \mathbf{s}_{k}^{H}(n)+\varepsilon \mathbf{I},
$$

where $\varepsilon$ is the diagonal loading parameter. Since this beamformer has snapshots generated by data compounded on the receive channels, we call this the data-compounded-onreceive MVDR beamformer (DCR-MVDR). The key difference between the DCR-MVDR and DCT-MVDR beamformers is that they are performed on different dimension of data matrix $\mathbf{X}(n)$. The diagram of the DCR-MVDR beamformer is showed in Fig. 1(e).

\section{IMAGING DATA AND RESULTS}

\section{A. Data Acquisition and Beamformer Implementation}

We demonstrate the beamformers on imaging data provided by the Plane-wave Imaging Challenge in Medical Ultrasound (PICMUS) [23]. The datasets were recorded using a Verasonics Vantage 256 research scanner and L11 probe (Verasonics Inc., Redmond, WA). The data also includes simulations of point-targets with Field II [28], [29] configured to model the same system. The probe is a 128-element linear array which has an element size of $0.27 \times 5.0 \mathrm{~mm}^{2}$, separated by a $0.03 \mathrm{~mm}$ kerf $(N=128)$. An excitation voltage of two and a half cycles is applied to generate an ultrasound pulse with a center frequency at $5.208 \mathrm{MHz}$ and a $67 \%$ pulse-echo bandwidth. The signals are sampled at 20.832 Msamples/s. Each set contains data acquired by 75 steered plane-waves distributed from $-16^{\circ}$ to $+16^{\circ}(M=75)$. All beamformers are performed on RF data to generate envelope images with a pixel size of $0.0739 \mathrm{~mm} \times 0.0986 \mathrm{~mm}$.

Beamformed images are generated using data from all 75 firing angles. In all spatial smoothing approximations, we set the subarray length $L$ equal to a half the size of the input vector. The diagonal loading parameter is set relative to $\Delta=$ $\operatorname{Tr}(\widehat{\mathbf{R}}) / L$, where $\widehat{\mathbf{R}}$ is the estimated matrix. It is selected using a trial-and-error method based on visualization of the generated image. In the double MVDR, we set $\varepsilon_{r, i}=\varepsilon_{t}=2 \Delta$. In the joint MVDR, $\varepsilon_{r}=0.1 \Delta$ for the receive and $\varepsilon_{2}=0.5 \Delta$ for the transmit. These diagonal loading parameters are applied to all datasets. In the PCM-MVDR, we set $\varepsilon=10 \Delta$ for data from the simulated point-targets and increase it to $\varepsilon=50 \Delta$ for the other datasets. For the DCT-MVDR and DCR-MVDR we use: $\varepsilon=\Delta$ for simulated point-target data and $\varepsilon=5 \Delta$ for the others. In these beamformers, $\Delta$ is the average of elements on the main diagonal of $\widehat{\mathbf{R}}_{\mathrm{PCM}}, \widehat{\mathbf{R}}_{\mathrm{DCT}}$ and $\widehat{\mathbf{R}}_{\mathrm{DCR}}$, respectively. In the four beamformers: DAS, PCM-MVDR, DCT-MVDR, and DCR-MVDR, the images are generated with an F-number of 1 . However, for the double and joint MVDR the images are generated without limiting the receive aperture. This helps maximize the spatial resolution for these beamformers.

\section{B. Evaluation Metrics}

We evaluate each beamformer based on the spatial and contrast resolution of the generated images. The spatial resolution is quantified using the responses of each beamformer to individual scatterers. Because all MVDR-based beamformers are developed based on an assumption of narrow-band signals that ignores temporal correlation, they mainly improve the lateral resolution. Thus, we are interested in the full width at half maximum (FWHM) of the response in the lateral direction only. The narrower the lateral FWHM the better the beamformer performance.

The contrast resolution is measured using the contrast ratio (CR) between a lesion and the background, given by [19]

$$
\mathrm{CR}=\frac{I_{\text {out }}-I_{\text {in }}}{\sqrt{I_{\text {out }}^{2}+I_{\text {in }}^{2}}},
$$

where $I_{\text {in }}$ and $I_{\text {out }}$ are the mean intensities (in decibels) measured inside and outside the lesion, respectively. The term $\mathrm{CR}$ has a value of 1 for perfect contrast, and a value of 0 for no contrast between the lesion and background. The background kernel is selected as a circular ring enclosing the lesion with an area that is the same as that of the lesion. This helps to minimise the effects of variations in the attenuation and diffraction of the ultrasound.

The eSNR calculation requires multiple RF datasets of the same scan which are not available, thus we do not measure it in this study. Another common metric to quantify the contrast resolution is the contrast-to-noise ratio (CNR). However, we 


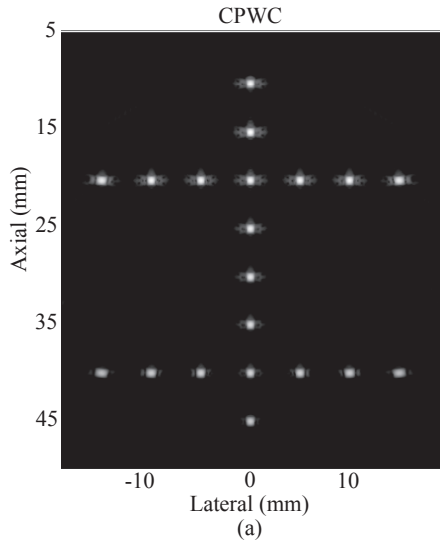

(a)

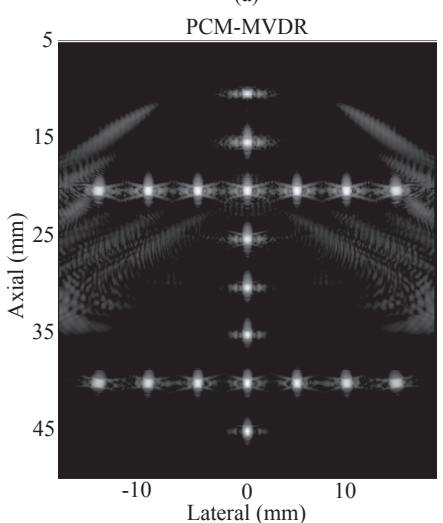

(d)

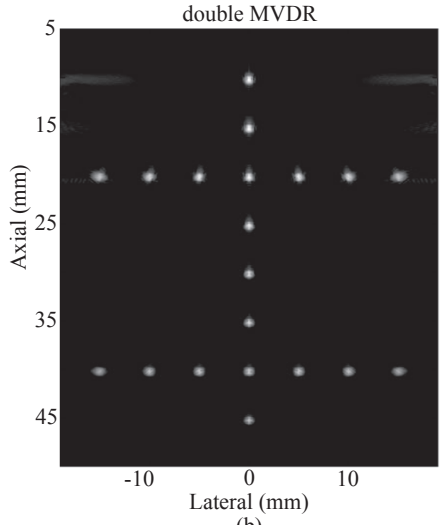

(b)

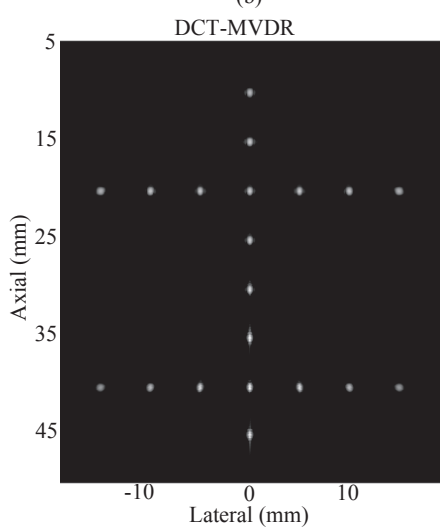

(e)

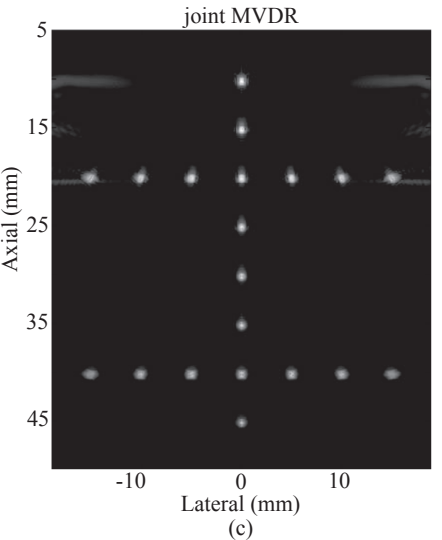

(c)

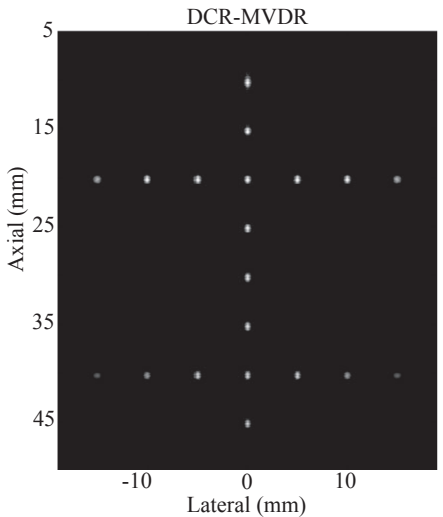

(f)

Fig. 2. Simulated images of 20 point-targets generated with different beamformers: (a) CPWC, (b) double MVDR, (c) joint MVDR, (d) PCM-MVDR, (e) DCT-MVDR, and (f) DCR-MVDR. All images are log-compressed and displayed with a dynamic range of $60 \mathrm{~dB}$.

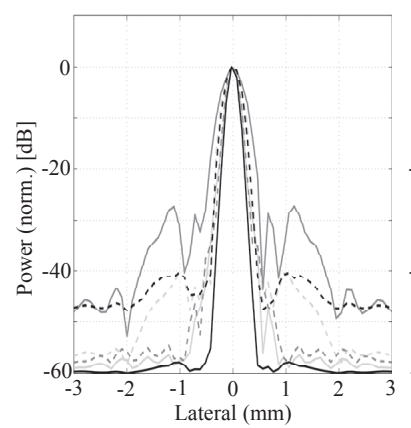

(a)

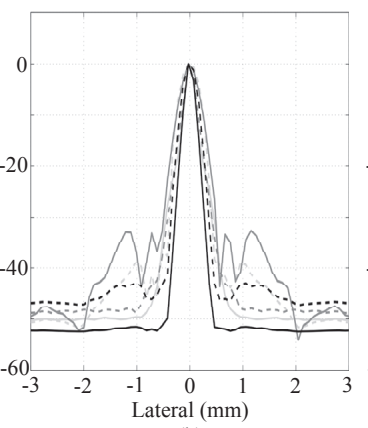

(b)

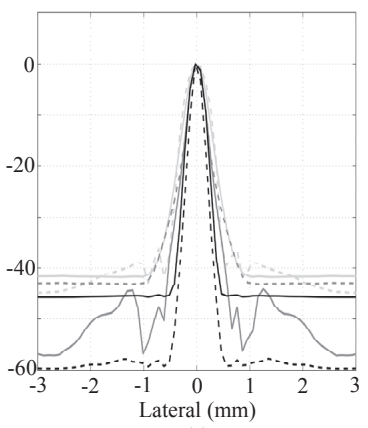

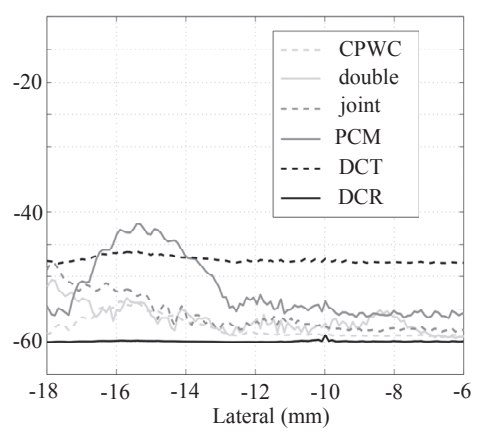

Fig. 3. Lateral responses to point-targets at different depths: (a) $15 \mathrm{~mm}$, (b) $30 \mathrm{~mm}$, and (c) $45 \mathrm{~mm}$. The beamformers tested are: CPWC (CPWC), double MVDR (double), joint MVDR (joint), PCM-MVDR (PCM), DCT-MVDR (DCT), and DCR-MVDR (DCR). To illustrate the artefacts shown on edges of the double, joint, and PCM-MVDR beamformed images, we extend the responses at depth $15 \mathrm{~mm}$ for each beamformer in (d). Plot (d) has a different $y$-axis scale from the others. The legend in plot (d) applies to all the other plots.

showed in previous studies that the CNR is inversely proportional to improvements in the spatial resolution [2], [12]. We found that any information about contrast that it provides was masked by a drop in CNR caused by improvements in spatial resolution. Therefore, it does not provide a useful independent metric with which to assess the performance of the MVDR beamformers.

\section{Simulated Point-Targets}

We first evaluate the beamformer performance on simulated data generated with 20 point-targets. Eight of them are located in the center of the image, ranged from $10 \mathrm{~mm}$ to $45 \mathrm{~mm}$ with a $5 \mathrm{~mm}$ separation. There are also two sets of 7 point-targets at depths of $20 \mathrm{~mm}$ and $40 \mathrm{~mm}$. In each of these sets, the points are evenly distributed from $-15 \mathrm{~mm}$ to $15 \mathrm{~mm}$ in the lateral direction.

Figures 2(a)-(f) show the images generated with the CPWC, double MVDR, joint MVDR, PCM-MVDR, DCT-MVDR, and DCR-MVDR beamformers, respectively. In the figures, the PCM-MVDR image has the most blurring point-targets, as well as the most artefacts in the area close to the transducer surface, i.e. the near-field region. Other MVDR-based images 

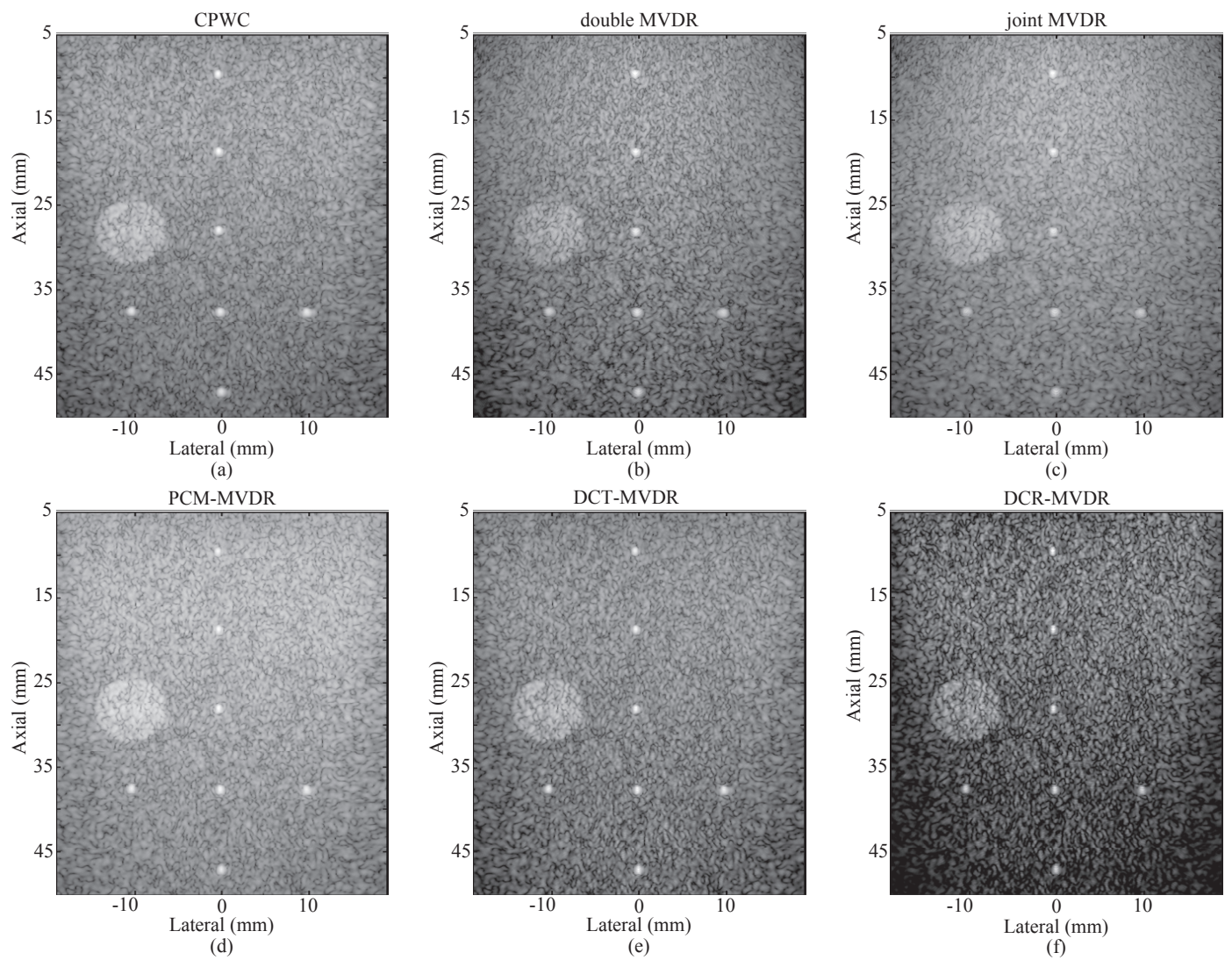

Fig. 4. Experimental images for resolution evaluation generated with different beamformers: (a) CPWC, (b) double MVDR, (c) joint MVDR, (d) PCM-MVDR, (e) DCT-MVDR, and (f) DCR-MVDR. All images are log-compressed and displayed with a dynamic range of $70 \mathrm{~dB}$.

show substantial improvements over DAS in term of the pointresolutions although the double and joint MVDR beamformers still exhibit some artefacts in the near-field. These artefacts are not observed in the DCT-MVDR and DCR-MVDR images. In the DCT-MVDR image, however, the point-resolution varies over the imaging depth. It is broad in the near-field but become much smaller in the far-field. Among the figures, the DCR-MVDR image has the best resolution as the points scatterers generate the most localised response at all depths. We quantify the image quality using the FWHMs measured through the mainlobes of the beamformer responses in Figs. 2. The averaged results are summarized in Table I.

To show the performance of the beamformers in more detail, we plot their lateral responses to some of the point-targets in Figs. 3(a)-(c). These are the responses to the central points at depths of $15 \mathrm{~mm}, 30 \mathrm{~mm}$, and $45 \mathrm{~mm}$, respectively. The plots show that all the MVDR-based algorithms, except the PCMMVDR, help reduce the mainlobes compared to CPWC. The plots also illustrate the variation of the mainlobe in the DCTMVDR response to a point-target. It has a mainlobe on a par with the CPWC in the near field, but becomes the smallest, even narrower than the DCR-MVDR, in the far-field.

To understand the artefacts that appear at the edges of each MVDR image, we plot the lateral profiles in this near-field region for all beamformers in Fig. 3(d) (at depth $10 \mathrm{~mm}$ ). The plot clearly reveals the strongest artefacts of the PCM-MVDR. Between the joint and double MVDR, the artefacts appearing on the joint MVDR image are slightly higher. The plot also shows the high-sidelobes of the DCT-MVDR response, which affect the imaging contrast in this region of the beamformer.

\section{Phantom Study}

We apply the beamformers to experimental data acquired by scanning a multi-purpose tissue-mimicking phantom (model 040GSE, CIRS, Norfolk, VA, USA) [23]. The manufacturer reported a sound speed of $1540 \pm 10 \mathrm{~m} / \mathrm{s}$ and a background attenuation coefficient slope of $0.5 \mathrm{~dB} \mathrm{~cm}^{-1} \mathrm{MHz}^{-1}$. The first dataset is designed to enable assessment of beamformer spatial resolution performance. The scanned volume includes seven nylon-monofilament wires, 100 microns in diameter, suspended against the speckle background. Five of them are located on the central axis and distributed from about $10 \mathrm{~mm}$ to $50 \mathrm{~mm}$ in depth. The other two are at a depth of $40 \mathrm{~mm}$, positioned at $-10 \mathrm{~mm}$ and $10 \mathrm{~mm}$ in the lateral direction.

The images generated with the different beamformers are shown in Figs. 4(a)-(f). Compared to the simulation, the MVDR-based images generated using the spatial smoothing approximation show less improvement over CPWC (in Fig. 4(a)). The resolutions are also reduced in the double and joint MVDR images (shown in Figs. 4(b) and (c)). Between these two, the joint MVDR image has lower resolution and a brighter appearance than the double MVDR, which indicates the lower impact of the MVDR beamformer as a spatial 


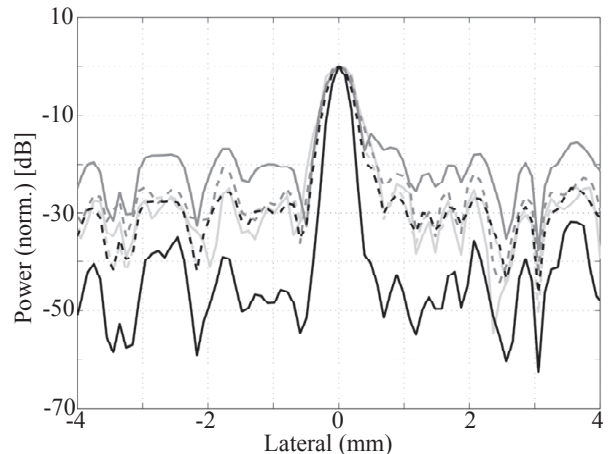

(a)

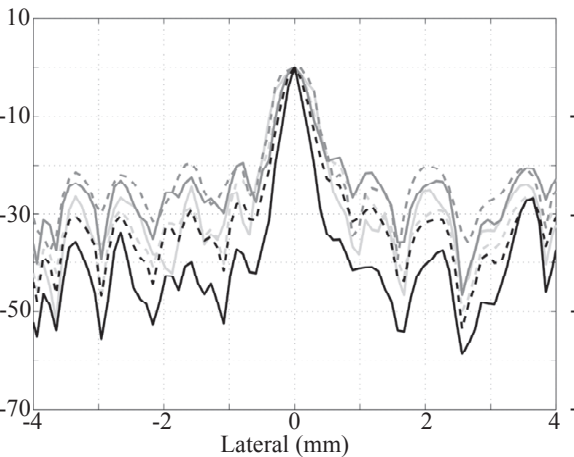

(b)

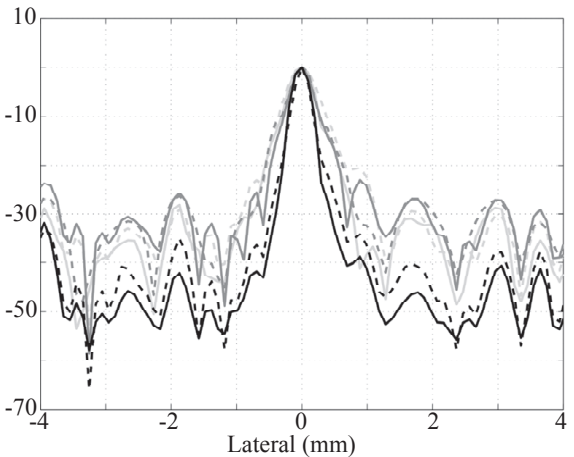

(c)

Fig. 5. Comparisons of the beam profile in cross-sections through the nylon-monofilament wires in the images shown in Figs. 4(a)-(f). The wire-targets are approximately at depths of: (a) $10 \mathrm{~mm}$, (b) $30 \mathrm{~mm}$ and (c) $50 \mathrm{~mm}$. The beam profiles are generated using CPWC (CPWC), double MVDR (double), joint MVDR (joint), PCM-MVDR (PCM), DCT-MVDR (DCT), and DCR-MVDR (DCR) beamformers. The legend in Fig. 3(d) applies to all these plots.
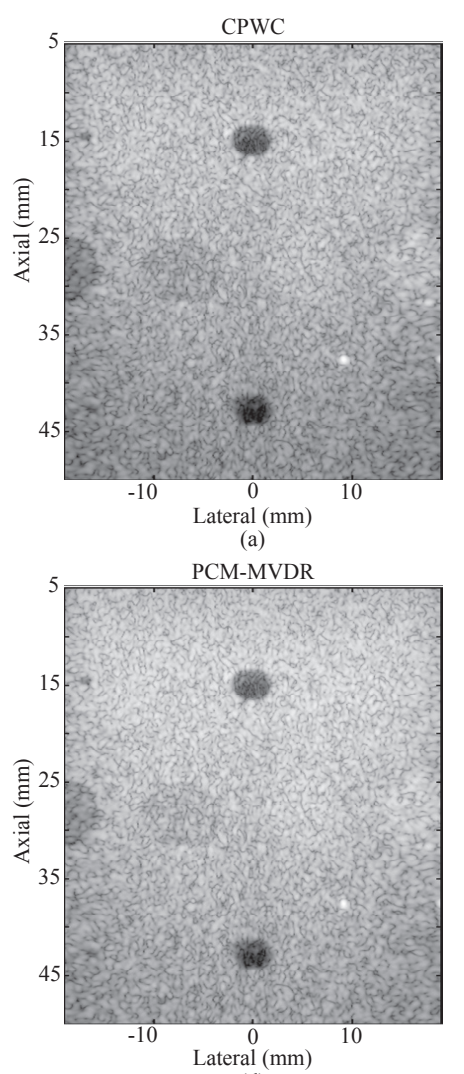

(d)

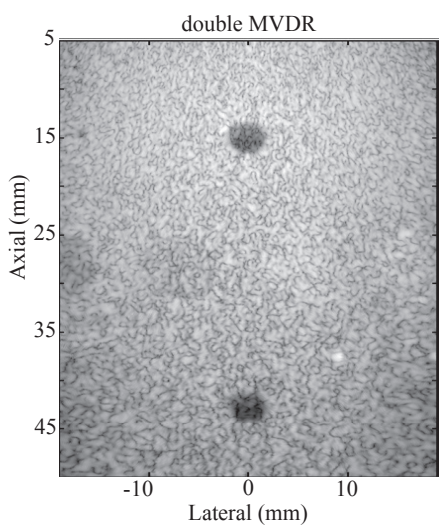

(b)

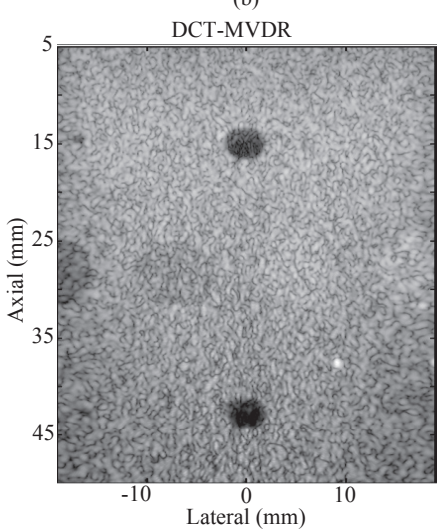

(e)
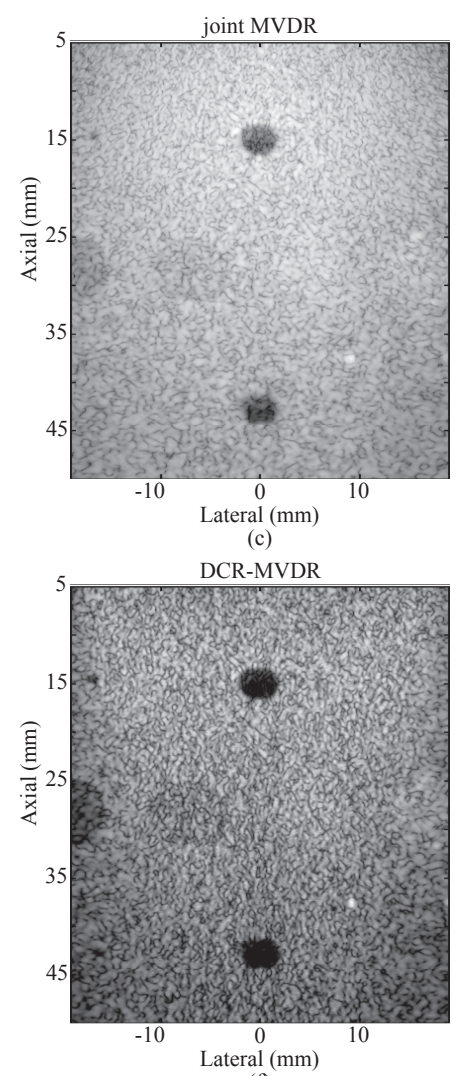

(f)

Fig. 6. Experimental images for contrast evaluation generated with the different beamformers: (a) CPWC, (b) double MVDR, (c) joint MVDR, (d) PCMMVDR, (e) DCT-MVDR, and (f) DCR-MVDR. All images are log-compressed and displayed with a dynamic range of $70 \mathrm{~dB}$.

TABLE I

PERFORMANCE METRICS MEASURED ON BEAMFORMED IMAGES FROM SIMULTATION, PHANTOM AND IN VIVO STUDIES

\begin{tabular}{|c|c|c|c|c|c|c|}
\hline \hline Beamformer & average FWHM (simulation) & average FWHM (experiment) & near-field CR & far-field CR & in-vivo CR & exec. time \\
\hline CPWC & $0.44 \pm 0.06 \mathrm{~mm}$ & $0.49 \pm 0.08 \mathrm{~mm}$ & 0.470 & 0.547 & 0.634 & $4 \mathrm{~min}$ \\
double MVDR & $0.27 \pm 0.10 \mathrm{~mm}$ & $0.52 \pm 0.12 \mathrm{~mm}$ & 0.369 & 0.454 & 0.549 & $420 \pm 5 \mathrm{~min}$ \\
joint MVDR & $0.29 \pm 0.10 \mathrm{~mm}$ & $0.54 \pm 0.09 \mathrm{~mm}$ & 0.408 & 0.474 & 0.506 & $435 \pm 5 \mathrm{~min}$ \\
PCM-MVDR & $0.45 \pm 0.20 \mathrm{~mm}$ & $0.44 \pm 0.06 \mathrm{~mm}$ & 0.410 & 0.467 & 0.523 & $44 \pm 1 \mathrm{~min}$ \\
DCT-MVDR & $0.33 \pm 0.17 \mathrm{~mm}$ & $0.33 \pm 0.13 \mathrm{~mm}$ & 0.567 & 0.683 & 0.600 & $44 \pm 1 \mathrm{~min}$ \\
DCR-MVPB & $0.23 \pm 0.08 \mathrm{~mm}$ & $0.30 \pm 0.04 \mathrm{~mm}$ & 0.840 & 0.884 & 0.797 & $44 \pm 1 \mathrm{~min}$ \\
\hline \hline
\end{tabular}




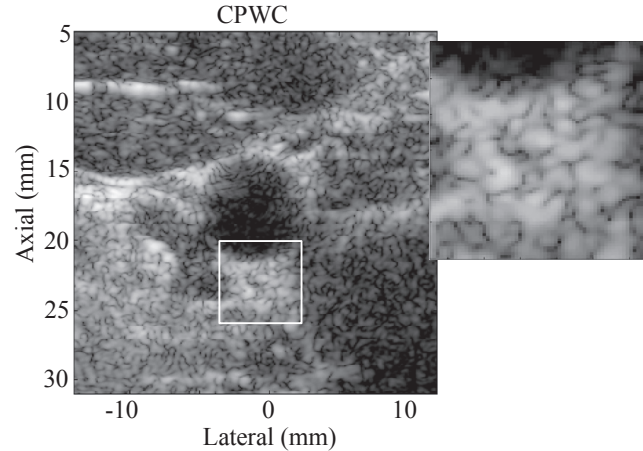

(a)

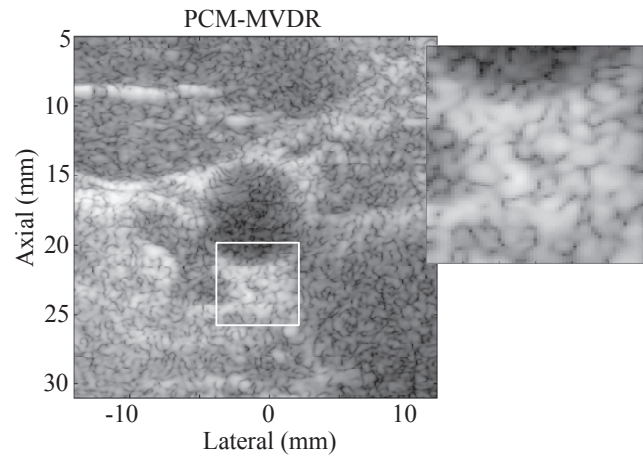

(d) double MVDR

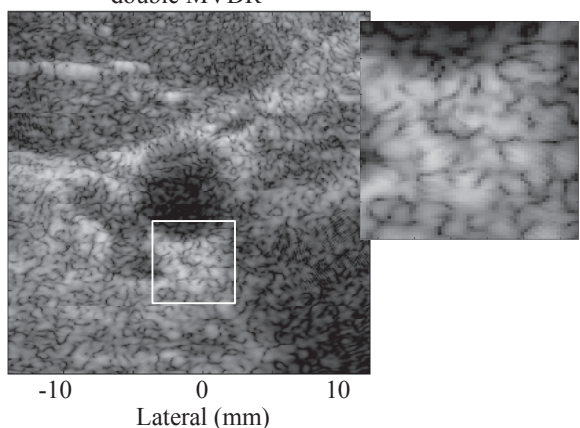

(b)

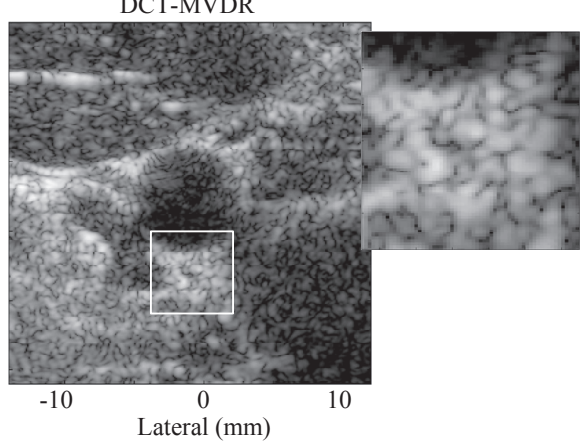

(e)

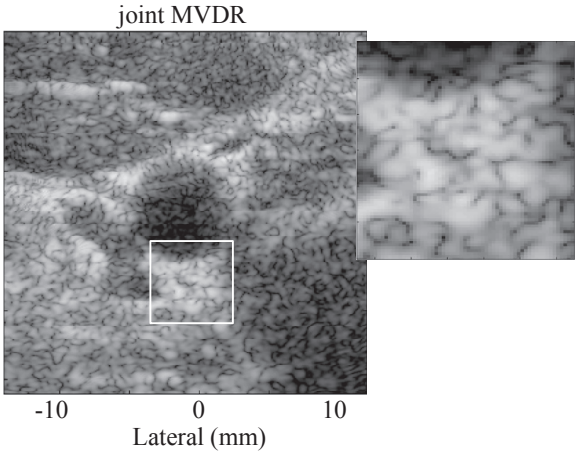

(c)

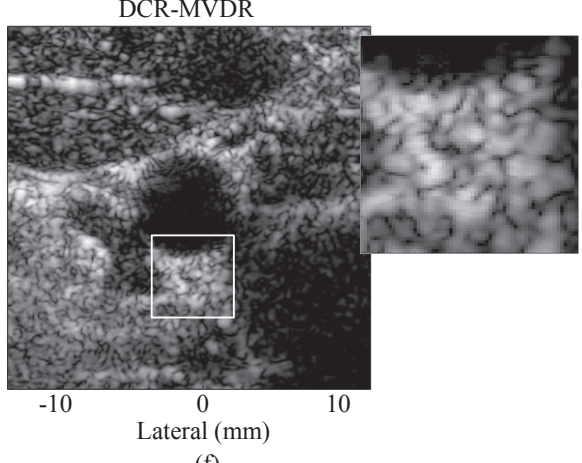

Fig. 7. Ultrasound images of the carotid (cross-sectional view) generated with different beamformers: (a) CPWC, (b) double MVDR, (c) joint MVDR, (d) PCM-MVDR, (e) DCT-MVDR, and (f) DCR-MVDR. All images are log-compressed and displayed with a dynamic range of $60 \mathrm{~dB}$. Each image is shown with a magnified view of the region enclosed by the white square.

filter. Compared to the joint and double MVDR, the PCMMVDR is shown to have better performance on experimental data. It has a line-target resolution slightly higher than that generated with CPWC. The two proposed beamformers, DCTMVDR and DCR-MVDR, still offer clear improvements over CPWC. Similar to the simulation, however, the DCT-MVDR (in Fig. 4(e)) exhibits variations in resolution from the nearfield to the far-field. The DCR-MVDR (in Fig. 4(f)) still has the best performance as the image shows the smallest wiretargets and finest speckle pattern in the background. For each beamformer, we measures the beam profiles over all seven wire-targets and report the averaged FWHMs in Table I.

As for the simulation, we plot some examples of lateral beam responses to the central point-targets in Figs. 5(a)(c). They show beamformer profiles at depths of $10 \mathrm{~mm}$, $30 \mathrm{~mm}$, and $50 \mathrm{~mm}$, respectively. The plots mainly reveal the differences between the DCT-MVDR and the DCR-MVDR beamformers. In the near-field (at depth $15 \mathrm{~mm}$ ), the DCTMVDR has a similar mainlobe to the other MVDR beamformers while its sidelobes are higher. In this region, the DCR-MVDR obviously has the best performance with the narrowest mainlobe and lowest sidelobes. In the far-field (at depth $50 \mathrm{~mm}$ ), the DCT-MVDR becomes on a par with the DCR-MVDR achieving equivalent mainlobes and almost the same level of sidelobes.

To assess the contrast resolution, we apply the beamformers to the second dataset of the phantom study, acquired by scanning two anechoic cysts against a speckle background.
The cysts are $3 \mathrm{~mm}$ in diameter, positioned at depths around $15 \mathrm{~mm}$ and $45 \mathrm{~mm}$. Images generated with the beamforming strategies are shown in Figs. 6(a)-(f). In these figures, the contrast ratios of the double, joint and PCM-MVDR images (in Figs. 6(b)-(d)) are lower than that of the CPWC image (in Fig. 6(a)). Improvements are only observed in the DCT-MVDR and DCR-MVDR images (Figs. 6(e)-(f)). Between them, the best anechoic contrast for both cysts is with the DCR-MVDR. We measure the CRs on each cysts (near-field CR and far-field CR) and summarize the results in Table I.

\section{E. In vivo Study}

To show the robustness of our new DCT-MVDR and DCRMVDR beamformers in clinical settings, we demonstrate them on an in vivo dataset, obtained from a carotid ultrasound scan of a healthy person. Compared to the simulation and phantom studies, the object has more complex structure and the beamformer performance may be more affected by phase aberrations. Fig. 7(a) shows the image generated with the CPWC method, which contains a cross-section of the carotid artery located at the center. Those generated using the MVDRbased algorithms are shown in Figs. 7(b)-(f). Under normal conditions, the artery is shown as a round structure on the sonogram in which the lumen is displayed as an anechoic region surrounded by the specular scattering of the artery wall. The contrast ratio of this lumen is calculated for each of the beamformers and presented in Table I. The image is displayed together with a magnified view of a highly echogenic region 
below the vessel (enclosed by the white square). This shows the speckle pattern of a diffuse scattering region which helps evaluate the spatial resolution.

The images generated with double, joint and PCM-MVDR are shown in Figs. 7(b)-(d). They all have lower CRs compared to that calculated on the CPWC image. The degradation is caused by the artefacts inside the lumen generated with each of these beamformers. On the magnified views corresponding these images, the speckle patterns are similar to that shown with the CPWC. The image generated with our new DCTMVDR (shown in Fig. 7(e)) offers finer speckle pattern on the magnified view compared to the CPWC. However, the contrast measured through CR is slightly lower. This may be explained by the fact that the region of interest is in the near-field, where the pulse-echo beam has been shown to have a broad mainlobe and higher sidelobes (see Figs. 3(a) and (d)). The DCT-MVDR image can produce better CR at larger depths as shown in the simulation and phantom study.

Consistent improvements over CPWC are only observed in the DCR-MVDR image (in Fig. 7(f)). It enhances the contrast over the entire imaging region and delivers the sharpest vessel boundary, which indicates the effect of MVDR beamforming as a spatial filter. It also generates images with clearer discrete speckle spots which are difficult to detect in the CPWC and other images. Inside the lumen, the artefacts generated by the other MVDR-based algorithms are no longer presented. This makes it appear the most uniformly anechoic and results in the highest $\mathrm{CR}$.

\section{DISCUSSION}

From the point-target simulation to experiments, there is a considerable reduction in performance of the joint and double MVDR beamformers using the spatial smoothing. This loss can be explained by the fact that the real data is more noisy and has a greater level of interference. It is thus less good for estimating the covariance matrix. In the spatial smoothing approach, the diagonal loading typically ranges from $0.01 \Delta$ to $0.2 \Delta$, where $\Delta$ is the average of elements on the main diagonal of the estimated covariance matrix [8]. In this study, however, the diagonal loading parameters for the joint and double MVDR are out of this range. Yet the images obtained with them are still unstable, indicated by the artefacts appearing in the near-field region (see Figs. 2(b,c)). These artefacts could be suppressed by using apodization or a larger diagonal loading parameter. Both techniques, however, further reduce the image resolution. The resolution could be slightly improved by using different combinations of subarray length and diagonal loading. Nevertheless, this is not a focus of our study.

The low performance of the double MVDR can be explained using the acoustic reciprocity theorem. In this composite algorithm, the transmit MVDR is applied to vector $\mathbf{z}(n)$, comprising signals from different transmits. According to the theorem, two components of vector $\mathbf{z}(n)$, e.g. $z_{i}(n)$ and $z_{j}(n)$, can be considered as data acquired from the same beam using an $N$-element linear array to transmit and receive in directions $i$ and $j$. The weight vectors $\mathbf{w}_{r, i}$ and $\mathbf{w}_{r, j}$, however, are equivalent to the apodization vectors applied to the $N$ element aperture to generate each of the signals. They should be identical to guarantee $z_{i}(n)$ and $z_{j}(n)$ are backscattered from the same insonified beam. Thus, the transmit MVDR only works according to its principles if the receive MVDR weight vector remains unchanged at all steering angles. A large diagonal loading helps all the $\mathbf{w}_{r, i}$ to converge to a uniform vector, as for the DAS algorithm. However, this reduces the benefit of using the MVDR approach.

The degradation of the joint MVDR performance can also be explained using our spatial coherence approach. In the beamformer expression $\mathbf{w}_{e}^{H} \mathbf{X}(n) \mathbf{w}_{r}$, the product $\mathbf{w}_{e}^{H} \mathbf{X}(n)$ can be considered as a data vector obtained from one transmit beam with apodization $\mathbf{w}_{e}$. The apodization changes the transmit beamshape and affects the spatial coherence among the receive signals [21]. As $\mathbf{w}_{r}$ is the coefficient weight of the spatial filter used to decorrelate this vector, it must be adapted to $\mathbf{w}_{e}$. Similarly through acoustic reciprocity, $\mathbf{w}_{e}$ must be adapted to $\mathbf{w}_{r}$. In the joint MVDR beamformer, however, they are both calculated based on the pre-summed data $\mathbf{X}(n)$, independently from each other. Thus, this calculation is not sufficient to perform the adaptive beamforming accurately. Finding a proper way to jointly calculate these weight vectors, however, is a complex problem and remains a challenge to address.

The three MVDR beamformers without spatial smoothing: PCM-MVDR, DCT-MVDR, and DCR-MVDR can be viewed as hybrid algorithms between DAS and MVDR beamforming. Although the PCM-MVDR has the lowest point-target resolution in simulation, its performance is on par to that of the joint and double MVDR on experimental and invivo data. To some extent, it shows the benefit of the DAS beamforming step that improves the coherence and eSNR of the input data, which is used in the subsequent MVDR algorithm. When applied to synthetic aperture imaging in [18], the pseudocovariance matrix was implemented with a calculated time delay varying within a bounded interval that takes phase aberration into account. This strategy may lead to further improvements for PCM-MVDR beamforming but is beyond the scope of the present study. Throughout all datasets, the DCT-MVDR is shown to offer significant improvements over the PCM-MVDR. These validate the advantages of using the new generated snapshots $\mathbf{p}_{k}(n)$. They have statistical properties similar to each other and close to that of the input vector $\mathbf{v}(n)$, which lead to better decorrelation of the MVDR beamforming as a spatial filter.

Both DCT-MVDR and DCR-MVDR use much higher diagonal loading parameters compared to the typical range used with spatial smoothing (from $0.01 \Delta$ to $0.2 \Delta$ ). This could be explained by the high correlation among the new snapshots ( $\mathbf{p}_{k}(n)$ for DCT-MVDR and $\mathbf{s}_{k}(n)$ for DCR-MVDR). As a result, the estimated matrices $\widehat{\mathbf{R}}_{\mathrm{DCT}}$ and $\widehat{\mathbf{R}}_{\mathrm{DCR}}$ (without diagonal loading) have low effective rank. This can be considered as a limitation of our new proposed methods. Yet, both DCT-MVDR and DCR-MVDR substantially enhance the image resolution compared to CPWC in all datasets. They also offer a ten-fold reduction in computational time compared to 
the double and joint MVDR beamformers. The computational time for each beamformer is presented in Table I. The most time consuming step in the double or joint MVDR relates to the subarray averaging. This requires scanning through each of the columns and rows of $\mathbf{X}(n)$. The execution times are all measured using Matlab (Mathworks Inc. Natick, MA, USA) on a desktop PC (Windows 7, 64-bit system, Intel $($ ) Core $^{\text {TM }}$ i7-4770, and $8 \mathrm{~Gb}$ Memory). Hence these figures are only approximate indications of the relative un-optimised computational load, distorted by the strengths and weaknesses of Matlab.

Compared to the DCT-MVDR, the DCR-MVDR has higher performance in the near-field region. The lower performance of the DCT-MVDR in that region mainly comes from the assumption that $\mathbf{v}(n)$ is generated from the focus of a 75element pseudo-phase-array, which requires an approximation of the plane-wave transmission as a spherical pulse. Several factors in the near-field region, such as diffraction [30], prevent the backscattered plane-wave from being approximated as a spherical wave propagated from one element of the pseudophase-array. In the far-field where this approximation become reasonable, the performance of the DCT-MVDR increases and become on par to that of the DCR-MVDR beamformer.

In our analytical framework, the new snapshots are generated based on an approximation of the covariance matrix to the spatial coherence among the input signals. Compared to the matrix estimation in (16) or (19), the standard approaches for estimation of spatial coherence often includes another step for temporal averaging [25], [26]. However, we can drop this step because of the narrow-band signals assumption. This implies that the echo signals extracted at different time instants have statistics that are independent from each other. In some other MVDR beamforming studies [19], the covariance matrix was estimated with a temporal averaging step. This estimation then becomes consistent with the exact definition of spatial coherence. To explore the effect of this step, we have added additional temporal averaging into our beamforming calculation for the first dataset of the phantom study. The covariance matrix is averaged over a time interval that relates to one wavelength in the axial direction. The generated images are smoother in the axial direction but there is no gain in lateral resolution. Furthermore, the temporal averaging increases the calculation excessively. Thus, we perform all our beamformer calculations at one time instance only and approximate them to the spatial coherence.

Both DCT-MVDR and DCR-MVDR beamformers were developed based on a feature of the CPWC whereby the entire linear array is used to capture the backscattered data in all transmits. Thus, they can be extended for use in synthetic aperture imaging or pixel-based beamforming if such algorithms share similar features in their data acquisitions. In our study, we calculate both DCT-MVDR and DCR-MVDR by assuming the sound-speed is constant. This could lower the beamformer performance because of phase abberation. It has been shown in other studies that the VCZ theorem can also be used to form an adaptive DAS beamformer [31]-[33]. This helps to restore some of the echo coherence lost when the wave speed or attenuation in the medium is unknown and spatially variable. Integrating this work into our spatial coherence approach for MVDR beamforming could enhance the image quality and remains for further investigation.

\section{SUMMARY}

We have proposed two new MVDR beamformers that are designed to integrate with coherent plane-wave compounding. In our study, the beamformers were developed in a framework where the MVDR beamforming is analyzed as a spatial filter and the correlation among echo signals is approximated by the spatial coherence, a concept from modern optics. From the analysis, we have drawn two MVDR beamformers that are applied to compounded data. The covariance matrix in each beamformer is estimated through slightly different combinations of the backscattered echoes. This helps us to avoid using the spatial smoothing approximation which reduces the image resolution. Our analysis also emphasizes the benefit of using DAS beamforming together with MVDR. It not only enhances the eSNR in the incoming signal but also improves the similarity among them before the MVDR decorrelation is applied.

Through demonstrations on imaging data, we show that our proposed methods offer significant improvements over CPWC in terms of both contrast and spatial resolution. They also outperform and are more computationally efficient than some other existing MVDR-based methods that require the spatial smoothing approach. By providing these beneficial features without compromising hardware complexity, the new beamformers show the importance of using a spatial-coherence approach to improve the efficacy of MVDR beamforming as two-way focussing systems become increasingly popular in ultrasound imaging applications.

\section{ACKNOWLEDGMENT}

We thank the PICMUS organizers, including Prof. Jørgen A. Jensen from the Technical University of Denmark; Dr. Alfonso Rodriguez-Molares from the Norwegian University of Science and Technology; Prof. Hervé Liebgott, Dr. Frederic Cervenansky, and Dr. Oliver Bernard from the University of Lyon for sharing imaging data and the code for coherent compounding. We are most grateful to the anonymous referees for their constructive comments and suggestions during the review process.

\section{REFERENCES}

[1] B.D. Van Veen and K.M. Buckley, "Beamforming: A versatile approach to spatial filtering," IEEE ASSP Mag., vol. 5, no. 2, pp. 424, 1988.

[2] N.Q. Nguyen and R.W. Prager, "Minimum variance approaches to ultrasound pixel-based beamforming," IEEE Trans Med Imag, vol. 36, no. 2, pp. 374-384, 2017.

[3] K. E. Thomenius, "Evolution of ultrasound beamformers," Proceedings of the IEEE Ultrasonics Symposium., pp. 1615-1622, 1996.

[4] M. Karaman, P.-C. Li, and M. O'Donnell, "Synthetic aperture imaging for small scale systems," in IEEE Trans Ultrason Ferroelec Freq Control, vol. 42, no. 3, pp. 429-442, 1995.

[5] J.A. Mann and W.F. Walker, "A constrained adaptive beamformer for medical ultrasound: Initial results," in Proceedings of the IEEE Ultrasonics Symposium, pp. 1807-1810, 2002.

[6] M. Sasso and C. Cohen-Bacrie, "Medical ultrasound imaging using the fully adaptive beamformer," in Proc. IEEE Int. Conf. Acoust. Speech Signal Processing, pp. 489-492, 2005. 
[7] F. Vignon and M.R. Burcher, "Capon beamforming in medical ultrasound imaging with focused beams," in IEEE Trans Ultrason Ferroelec Freq Control, vol. 55, no. 3, pp. 619-628, 2005.

[8] J.-F. Synnevåg, A. Austeng, and S. Holm, "Adaptive beamforming applied to medical ultrasound imaging," IEEE Transactions on Ultrasonics, Ferroelectrics, and Frequency Control, vol. 54, no. 8, pp. 1606-1613, 2007.

[9] T.-J. Shan, M. Wax, and T. Kailath, "On spatial smoothing for directionof-arrival estimation of coherent signals," IEEE Transactions on Acoustics, Speech, and Signal Processing, vol. 33, no. 4, pp. 806-811, 1985.

[10] J.A. Jensen, S.I. Nikolov, K.L. Gammelmark, and M.H. Pedersen, "Synthetic aperture ultrasound imaging," in Ultrasonics, vol. 44, suppl., pp. e5-e15, 2006.

[11] G. Montaldo, M. Tanter, J. Bercoff, N. Benech, and M. Fink, "Coherent plane-wave compounding for very high frame rate ultrasonography and transient elastography" in IEEE Trans Ultrason Ferroelec Freq Control, vol. 56, no. 3, pp. 489-506, 2009.

[12] N.Q. Nguyen and R.W. Prager, "High-resolution ultrasound imaging with unified pixel-based beamforming," IEEE Trans Med Imag, vol. 35, no. 1, pp. 98-108, 2016.

[13] N.Q. Nguyen and R.W. Prager, "Ultrasound pixel-based beamforming with phase alignments of focused beams," IEEE Trans Ultrason Ferroelec Freq Control, vol. 64, no. 6, pp. 937-946, 2017.

[14] I.K. Holfort, A. Austeng, J.F. Synnevåg, S. Holm, F. Gran, and J.A. Jensen,"Adaptive receive and transmit apodization for synthetic aperture ultrasound imaging," in Proceedings of the IEEE Ultrasonics Symposium, pp. 1-4, 2009.

[15] A. Austeng, C.-I.C. Nilsen, A.C. Jensen, S.P. Näsholm, and S. Holm, "Coherent plane-wave compounding and minimum variance beamforming," in Proceedings of the IEEE Ultrasonics Symposium, pp. 24482451, 2011.

[16] O.M.H Rindal and A. Austeng, "Double adaptive plane-wave imaging," in Proceedings of the IEEE Ultrasonics Symposium, pp. 1-4, 2016.

[17] J. Zhao, Y. Wang, X. Zeng, J. Yu, B.Y.S Yiu, and A.C.H. Yu, "Plane wave compounding based on a joint transmitting-receiving adaptive beamformer," IEEE Transactions on Ultrasonics, Ferroelectrics, and Frequency Control, vol. 62, no. 8, pp. 1440-1452, 2015.

[18] Z. Wang, J. Li, and R. Wu, "Time-delay- and time-reversal-based robust Capon beamformers for ultrasound imaging," IEEE Transactions on Medical Imaging, vol. 24, no. 10 pp. 1308-1322, 2005.

[19] S. Mehdizadeh, A. Austeng, T.F. Johansen, and S. Holm, "Eigenspace based minimum variance beamforming applied to ultrasound imaging of acoustically hard tissues," IEEE Trans Med Imag, vol. 31, no. 10, pp. 1912-1921, 2012.

[20] S.A. Vorobyov, "Adaptive and robust beamforming," in Academic Press Library in Signal Processing: Array and Statistical Signal Processing, vol. 3, R. Chellapa and S. Theodoridis, Eds. San Diego, CA, USA: Academic, 2014, pp. 503-522.

[21] M.A. Lediju, G.E. Trahey, B.C. Byram, and J.J. Dahl, "Short-lag spatial coherence of backscattered echoes: Imaging characteristics," in IEEE Trans Ultrason Ferroelec Freq Control, vol. 58, no. 7, pp. 1377-1388, 2011.

[22] R. Mallart and M. Fink, "The van Cittert-Zernike theorem in pulse echo measurements," J Acoust Soc Am, vol. 90, no. 5, pp. 2718-2727, 1991.

[23] H. Liebgott, A. Rodriguez-Molares, F. Cervenansky, J.A. Jensen, and O. Bernard, "Plane-wave imaging challenge in medical ultrasound," in Proceedings of the IEEE Ultrasonics Symposium, pp. 1-4, 2016.

[24] M. Viberg, "Introduction to array processing," in Academic Press Library in Signal Processing: Array and Statistical Signal Processing, vol. 3, R. Chellapa and S. Theodoridis, Eds. San Diego, CA, USA: Academic, 2014, pp. 463-502.

[25] J.W. Goodman, Statistical optics. New York: Wiley-Interscience, 2000.

[26] A.R. Thompson, J.M. Moran, and G.W. Swenson, Interferometry and synthesis in radioastronomy, 2nd ed. New York, NY: Wiley, 2001.

[27] N. Bottenus and K.F. Üstüner, "Acoustic reciprocity of spatial coherence in ultrasound imaging," in IEEE Trans Ultrason Ferroelec Freq Control, vol. 62, no. 5, pp. 852-861, 2015.

[28] J.A. Jensen and N.B. Svendsen, "Calculation of pressure fields from arbitrarily shaped, apodized, and excited ultrasound transducers," IEEE Trans Ultrason Ferroelec Freq Control, vol. 39, no. 2, pp. 262-267, 1992.

[29] J.A. Jensen, "Field: A program for simulating ultrasound systems," Med Biol Eng Comp, vol. 34, Supplement 1, Part 1, pp. 351-353, 1996.

[30] T.L. Szabo, Diagnostic ultrasound imaging: inside out, 2nd ed. Boston, MA: Elsevier Science, 2014.
[31] M. O'Donnell and S.W. Flax, "Phase-aberration correction using signals from point reflectors and diffuse scatterers: measurements," IEEE Trans Ultrason Ferroelectr Freq Control, vol. 35, no. 6, pp. 768-774, 1988.

[32] L. Nock, G.E. Trahey, and S.W. Smith, "Phase aberration correction in medical ultrasound using speckle brightness as a quality factor," $J$ Acoust Soc Am, vol. 85, no. 5, pp. 1819-1833, 1989.1

[33] R. Mallart and M. Fink, "Adaptive focusing in scattering media through sound speed inhomogeneities: the van Cittert-Zernike approach and focusing criterion," J Acoust Soc Am, vol. 96, no. 6, pp. 3721-3732, 1994. 